incorrect equipment could have had major consequences. If a rubber dam

$\varangle$ and Luer Lock syringe were not available it may have been safer to simply access the pulp chamber of the tooth and provide a sedative dressing until the tooth could be removed.

R. Long, Cardiff, UK

https://doi.org/10.1038/s41415-020-1439-4

\section{From IDB to ITU}

Sir, we discuss a case of a 74-year-old female presenting ten days post extraction of her lower left last standing molar to the emergency department with an extremely large left sided facial swelling. Upon taking a history it became apparent that the swelling developed almost immediately and did not change in size over the following ten days. The patient reported that the extraction needed a large volume of anaesthetic, but that there was no swelling prior to the extraction. She had re-presented to her GDP on the tenth day and was referred into our unit. She is a type 2 diabetic.

On presentation she had a large and firm left sided facial swelling, in the distribution of her left parotid gland. She had severe trismus, raised inflammatory markers (C Reactive Protein 312, White Cell Count 24.24, Neutrophils 21.79), and was showing early signs of sepsis. The extraction socket was healing.

Cross sectional imaging revealed a large collection in the left masseteric and parotid spaces, with tracking into the left parapharyngeal space and distortion of her oropharynx. Nasal endoscopy revealed swelling of her vocal cords.

A diagnosis of an infected haematoma was made, likely developing from a haematoma related to the inferior dental block.

The patient required surgical drainage in theatre, with large volumes of pus drained and due to airway swelling spent 12 hours intubated in the intensive care unit. She spent a further five days in hospital for intravenous antibiotic therapy.

We want to raise awareness of haematomas as a possible side effect of inferior dental blocks, and possible serious consequences. A haematoma is caused by rupture of blood vessels and consequential bleeding into the tissue spaces. The risk is reduced with negative aspiration to avoid intra vascular injection, slow delivery of local anaesthetic and knowledge of vessel anatomy. Swelling immediately post-delivery of anaesthetic is likely to be a haematoma, as swelling related to the surgery develops over subsequent days. Clinical review is required as they can become infected and require antibiotics.

Fortunately, they rarely occur but recognition of this possible complication and management is required, in order to reduce morbidity to the patient.

C. A. Richards, Bristol, UK

https://doi.org/10.1038/s41415-020-1440-y

\section{Dental radiography}

\section{Dens evaginatus}

Sir, working in the radiology department of a dental hospital, we recently encountered a case of an upper central incisor with a tubercular shaped projection from the palatal surface, in keeping with dens evaginatus: a developmental anomaly with a prevalence of $1-4 \%$. It presents as an accessory cusp consisting of enamel, dentine and pulp. Common sites are the occlusal surfaces of premolars and the lingual surfaces of anterior teeth. ${ }^{1}$

There is a risk of fracture from occlusal trauma, resulting in exposure of the pulp and devitalisation of the tooth. Early detection and treatment is therefore important with options often including enameloplasty. ${ }^{2}$ This is dependent on the presence, position and extension of

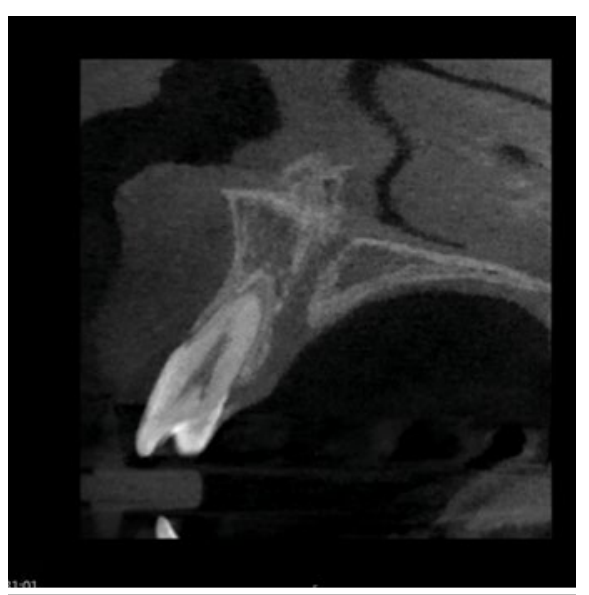

Fig. 1 CBCT image showing dens evaginatus 11 with a pulpal projection into the accessory cusp pulp tissue into the accessory cusp. Conventional images are not capable of identifying this with any level of accuracy. CBCT is an accessible, reliable and relatively low dose $3 \mathrm{D}$ modality, used here to show a significantly sized additional palatal cusp with a prominent pulp projection from the pulp of the 11 (Fig. 1). ${ }^{3}$

$3 \mathrm{D}$ imaging provides accurate assessment of a simple but important developmental anomaly with low radiation burden. It is essential that users of $\mathrm{CBCT}$ are adequately trained to interpret these images.

\section{B. Pritchard, F. A. Tefaghi, J. Makdissi,} London, UK

\section{References}

1. Ayer A, Vikram M, Suwal P. Dens evaginatus: a problem-based approach. Case Rep Dent 2015: 2015: 393209

2. Wright J T, Meyer B D. Anomalies of the developing dentition. In Nowak A J, Christensen J R, Mabry T R, Townsend J A, Wells M H. Pediatric dentistry infancy through adolescence, $6^{\text {th }}$ ed. pp 50-65. Philadelphia: Elsevier, 2019.

3. HPA Working party on dental cone beam CT equipment. HPA-CRCE-010: guidance on the safe use of dental cone beam CT (computed tomography) equipment, 2010.

https://doi.org/10.1038/s41415-020-1441-x

\section{OMFS}

\section{Oral ulceration with bony sequestration}

Sir, oral ulceration is a common condition with a range of aetiologies. A case of oral ulceration with development of osteitis and sequestrum from the overlying ulcer (OUBS) is reported from primary care. A 55-year-old caucasian male attended for a routine examination with a two week history of mild discomfort in the right posterior mandible. The patient had recently undergone emergency surgery to relieve pressure from cervical spine stenosis diagnosed as causing foot drop. Intraoral examination revealed a large ulcer with red inflamed irregular margins on attached gingiva of lingual shelf adjacent to 47 resembling an aphthous ulcer. However, the ulcer surrounded a $6 \times 4 \mathrm{~mm}$ attached whitish rough bony-hard disc (Fig. 1). Saline and chlorhexidine mouthwashes were prescribed and at a review appointment the sequestrated bone detached easily and healing was uneventful, 